\title{
Simultaneous Measurement of Strain and Temperature Using a Single Emission Line
}

\author{
Daniel Leandro, Martin Ams, Manuel Lopez-Amo Senior Member, IEEE, Tong Sun and Kenneth T.V. \\ Grattan.
}

\begin{abstract}
In this work, we present and demonstrate a novel sensor system for simultaneous measurement of strain and temperature through a unique combination of a long period grating (LPG) and a fiber laser based on a fiber Bragg grating (FBG). In order to achieve this, a new erbium doped fiber laser (EDFL) structure is created, showing an optical signal-to-noise ratio (OSNR) of $55 \mathrm{~dB}$ and a peak power measured on the OSA between -5 and $0 \mathrm{dBm}$. The strain and the temperature information can be obtained by using a unique emission line through monitoring both the fiber laser wavelength shift and the change of the power level, both of which showing a clear linear behavior.
\end{abstract}

Index Terms - Erbium lasers, fiber Bragg gratings, fiber lasers, long period gratings, LPG coupling.

\section{INTRODUCTION}

$\mathrm{F}$ IBER Bragg gratings (FBGs) have played an important role in communication systems, fiber lasers and optical fiber sensing since the 1980s. They have been widely used as optical fiber sensors due to their advantages demonstrated over their electrical counterparts, such as immunity to electromagnetic interference, compactness, multiplexing capability, resistance to harsh environments and low cost. As a result, FBGs have been widely used for structural health monitoring, oil pipe leak detection and gas detection [1]. Some applications have involved the use of FBGs to form laserbased sensor systems, taking full advantage of the narrow spectra of FBGs which are suitable for laser wavelength selection [2]. Compared to FBG-based sensor systems, these fiber laser-based sensor systems have shown many advantages including a higher resolution for wavelength shift identification, higher OSNR and enhanced capability for remote sensing. As an example a fiber laser-based sensor can be used to monitor as far as $200 \mathrm{~km}$ without any external amplification [3], in contrast, a FBG-based sensor illuminated by a broadband light source can only reach a maximum

Manuscript received August, 29, 2014. This work was supported by the by the Spanish Government projects TEC2010-20224-C02-01, TEC2013-47264C2-2-R, the FEDER funds and by the European COST action TD1001.

D. Leandro and M. Lopez-Amo, are with Department of Electric and Electronic Engineering, Public University of Navarra, Campus Arrosadia S/N, 31006, Navarra, Spain. (e-mail: daniel.leandro@unavarra.es).

M. Ams, T. Sun and K. T. V. Grattan, are with the School of Engineering and Mathematical Sciences, City University London, London EC1V, UK.

Copyright (c) 2013 IEEE. Personal use of this material is permitted. distance up to $25 \mathrm{~km}$, limited by Raylegh Scattering [4].

A common issue of using FBGs as sensing devices is the cross-sensitivity between the measurands, i.e. strain and temperature. A number of techniques have been reported in order to overcome this limitation and thus to achieve simultaneous measurement of strain and temperature. These systems include those using photonic crystal fibers [5] and multimode fibers [6], in combination with FBGs [7] or LPGs [8] among others. Most of these systems, however, have a low resolution and are fragile showing some difficulty in splicing or handling if a microstructured fiber is used. Other techniques include the use of specifically designed fiber Bragg gratings (FBGs) [9], [10] or gratings written into special fibers [11] or the combination of a grating and some other physical property like the dependence of the modes beating frequency on the laser cavity [12]. Few papers have been published considering both the simultaneous measurement and fiber lasers. These schemes have interesting features, for example, a high signalto-noise ratio would allow the sensors to be interrogated over tens of kilometers [12], [13].

In this paper we present a new sensor system based on an erbium doped fiber laser involving a FBG and coupled with a LPG, for simultaneous measurement of strain and temperature. Both parameters can be simultaneously measured, because they induce both a fiber laser wavelength shift and a change of the power level coupled out by the LPG. The utilization of a unique emission line for measuring both parameters allows a more efficient use of the optical spectrum, which is an important factor for sensor multiplexing using WDM techniques [14].

\section{PRINCIPLE OF OPERATION}

The experimental setup is shown in Fig. 1. The laser gain medium is a $0.7 \mathrm{~m}$ erbium doped fiber (EDF) Er110, provided by Liekki and pumped by a $980 \mathrm{~nm}$ laser with a maximum output power of $500 \mathrm{~mW}$. This fiber was chosen due to its flat gain profile around $1535-1540 \mathrm{~nm}$ and the high gain provided.

As shown in Fig.1, a wavelength division multiplexer (WDM) couples the $980 \mathrm{~nm}$ pump-light into the linear laser cavity, with a FBG being connected to the other end of the erbium doped fiber. The FBG also acts as a temperature/strain sensor in this work. The FBG studied is centered at 1536-1537 $\mathrm{nm}$ with a reflectivity higher than $95 \%$ and the free termination was immersed in a refractive-index-matching gel to avoid undesired reflections. The reflected laser light travels back through the WDM and a polarization controller (PC) to 
the LPG where the signal is partially attenuated due to the mode coupling between the fiber core and the cladding of the LPG. The PC is included in the system to minimize the polarization dependence of the LPG, which was induced by the UV exposure during the fabrication process in a nonpolarization-maintaining fiber [15]. This polarization dependence may be eliminated by writing the grating on photosensitive PM-fiber.

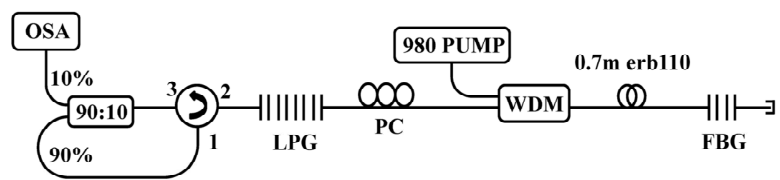

Fig. 1. Schematic diagram of the experimental setup.

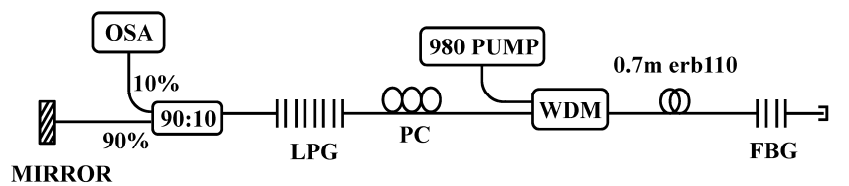

Fig. 2. Scheme of the experimental setup using a mirror instead of a circulator.

Finally, as indicated in Fig. 1, a circulator is used to couple the light into the cavity and to extract a $10 \%$ of the power to be monitored by an optical spectrum analyzer (OSA). The circulator shown in Fig.1 can be replaced by a silver-coated fiber optic mirror as illustrated in Fig. 2. Both options have been analyzed in this work showing similar results. Therefore, the first scheme was used to demonstrate the proof of concept.

The LPG used in the setup is centered at $1532 \mathrm{~nm}$ and both the FBGs and the LPGs were fabricated at City University London. The grating wavelengths were chosen in order to place the spectral profile of the signal reflected by the FBG at the linear slope of the LPG. In this way when the LPG shape shift occurs due to a temperature/strain change the amplitude of the laser varies linearly. Figure 3 shows the superimposed optical spectra of the LPG and FBG. As can be seen in the figure, the reflected peak of the FBG is placed at the slope of the LPG.

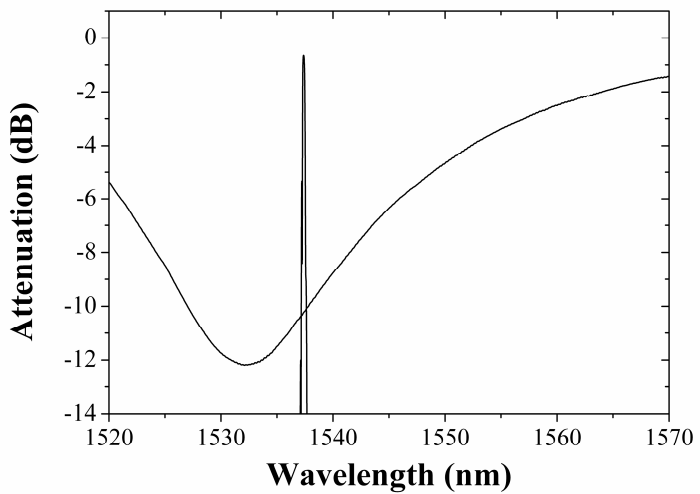

Fig. 3. Superimposed optical transmitted and reflected spectra of LPG and FBG respectively.
In order to validate the sensing technique a series of experiments has been undertaken. Even though the system is designed to discriminate temperature from strain when both FBG and LPG were subject to the same strain and temperature conditions, there is, however, some difficulty in performing these measurements thus in this work an indirect method is used for verification of the sensing principle proposed. First of all, the sensitivities of the wavelength and peak power of the laser to strain and temperature were measured independently. Afterwards the proper behavior of the laser was verified when both sensors are subjected to changes simultaneously. Finally the equations to obtain $\Delta \varepsilon$ and $\Delta T$ from the wavelength and the peak power of the emission line are described.

\section{EXPERIMENTAL VERIFICATION}

Before setting up the fiber laser, in order to obtain an optimal wavelength value for the FBG, a study of the attenuation given by the LPG at different FBG wavelengths was carried out as a function of the temperature. To perform the study a LPG centered at $1532 \mathrm{~nm}$ was heated in a climatic chamber from 30 to $100{ }^{\circ} \mathrm{C}$. The attenuation given by the LPG was measured at different wavelengths every $10^{\circ} \mathrm{C}$ (Fig. 4) This study shows that a higher dynamic range and sensitivity can be achieved by using a FBG with a central wavelength situated at around $1536-1537 \mathrm{~nm}$ as indicated in Fig.4. Thus these were the wavelengths chosen for the FBGs fabricated for this work. In addition to the sensitivity discussed, the sensing range has also been considered by carefully locating the Bragg wavelength of the FBG within the linear slope of the LPG thus to optimize both the sensitivity and sensing range.

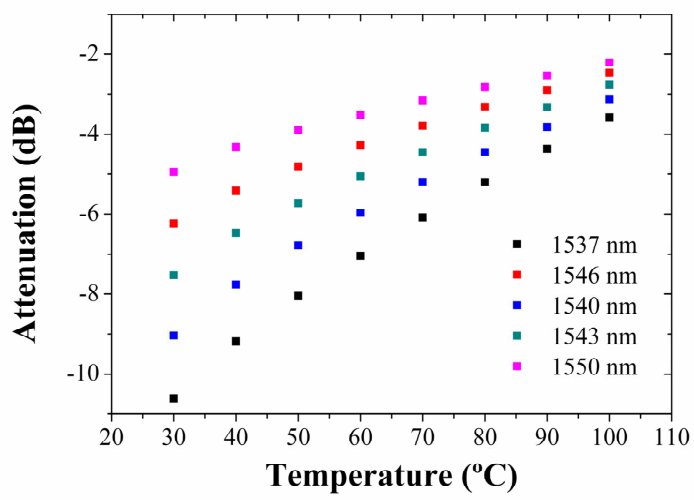

Fig 4. Relationship between temperature and transmitted power of the LPG for different FBG wavelengths

By using the set-up shown in Fig.1, the laser output can be measured on the OSA as shown in Fig.5. The properties of the EDFL were studied resulting in an optical signal-to-noise ratio that is higher than $55 \mathrm{~dB}$ and a peak power measured on the OSA between -5 and $0 \mathrm{dBm}$. This peak power coupled out of the LPG, however, depends on the temperature and strain of the LPG although its wavelength is still dependent both on the temperature and the strain applied to the FBG.

Four tests were undertaken in order to obtain the wavelength and the peak power response of the system to temperature and strain variations applied to one sensor independently each time. For the strain tests, the gratings were glued to a translation stage which allowed the strain to be set 
with an error of $\pm 6 \mu \varepsilon$. The temperature tests were performed in a climatic chamber with a resolution of $\pm 0.1^{\circ} \mathrm{C}$.

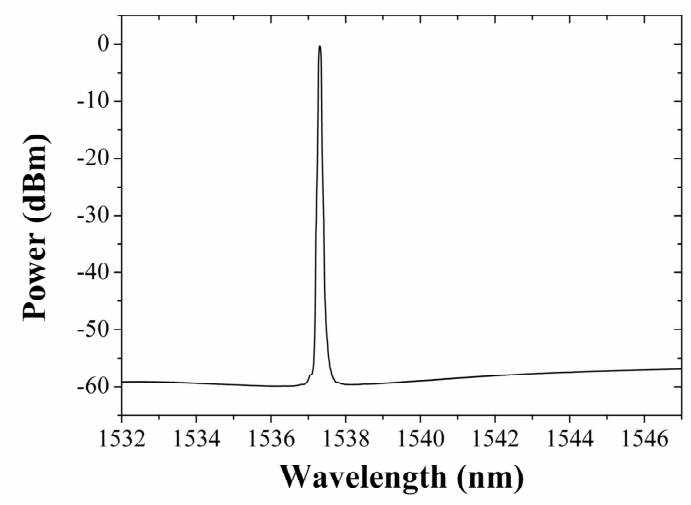

Fig. 5. Spectrum of the laser output signal on the OSA.

The first two tests studied the dependence of the laser wavelength as a function of the strain and temperature variation surrounding the FBG. The FBG was heated in a climatic chamber while the LPG was kept at room temperature. No strain was applied to any of them. The response is clearly linear with a typical sensitivity $k_{\lambda T}=8.97$ $\mathrm{pm} /{ }^{\circ} \mathrm{C}$ with a square of the correlation coefficient $\mathrm{R}^{2}=0.997$ (Fig. 6). Similarly a strain sweep was conducted to the FBG while the LPG remained steady. Both of them were kept at room temperature. The strain sensitivity from the FBG had a linear behavior with a sensitivity $k_{\lambda \varepsilon}=0.824 \mathrm{pm} / \mu \varepsilon$ with a $\mathrm{R}^{2}=0.999$ (Fig. 7).

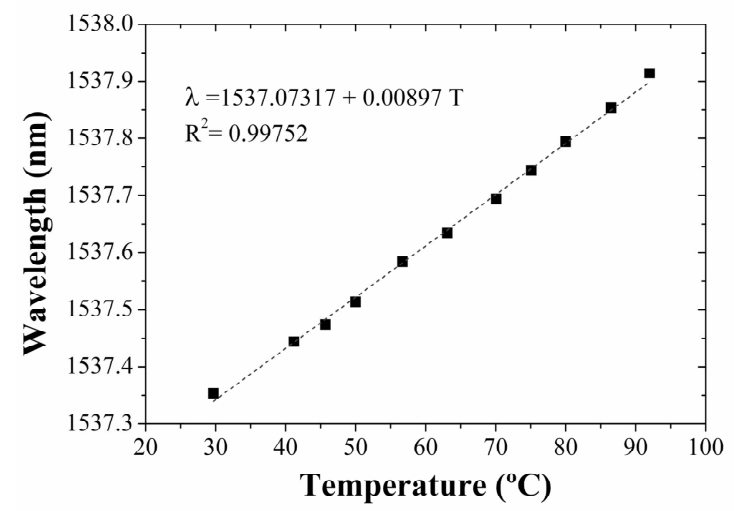

Fig. 6. Relationship between the fiber laser wavelength and temperature applied to the FBG (LPG was kept steady at room temperature).

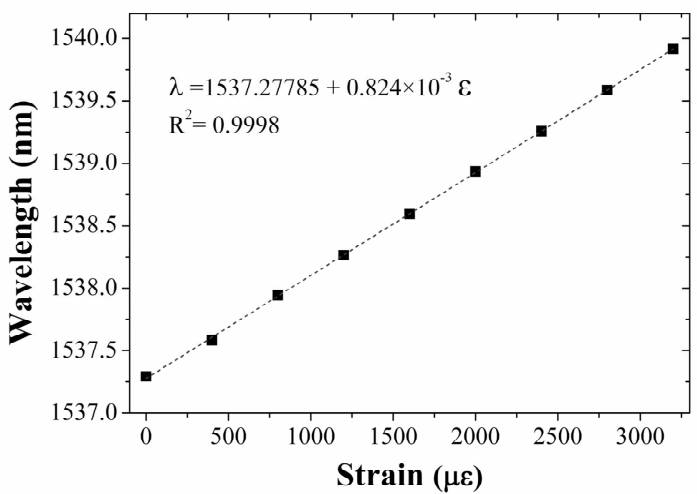

Fig. 7. Relationship between the fiber laser wavelength and strain applied to the FBG (LPG was kept steady at room temperature).
In the same manner, two tests were carried out to study the changes on the peak power of the laser caused by the variation of temperature and strain applied on the LPG. In the first test the LPG was heated in the climate chamber while the FBG was kept at room temperature (both of them suffered no strain). Again a linear behavior can be observed with a power sensitivity $k_{P T}=0.0112 \mathrm{~mW} /{ }^{\circ} \mathrm{C}$ with a $\mathrm{R}^{2}=0.998$ (Fig. 8). In the last experiment the LPG was placed on the translation stage where a strain sweep was performed. Meanwhile the FBG remained steady and both of them were kept at room temperature. As shown in Fig. 9 the sensitivity measured was $k_{P_{\varepsilon}}=-3.09 \times 10^{-5} \mathrm{~mW} / \mu \varepsilon, \mathrm{R}^{2}=0.994$.

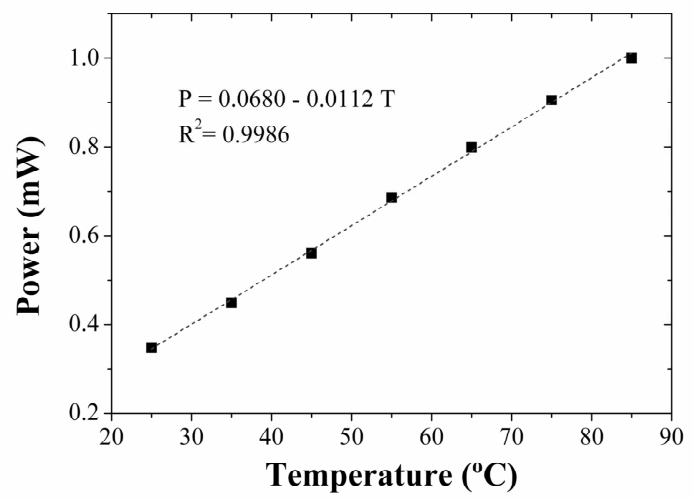

Fig. 8. Relationship between the fiber laser peak power and temperature applied to the LPG (FBG was kept steady at room temperature).

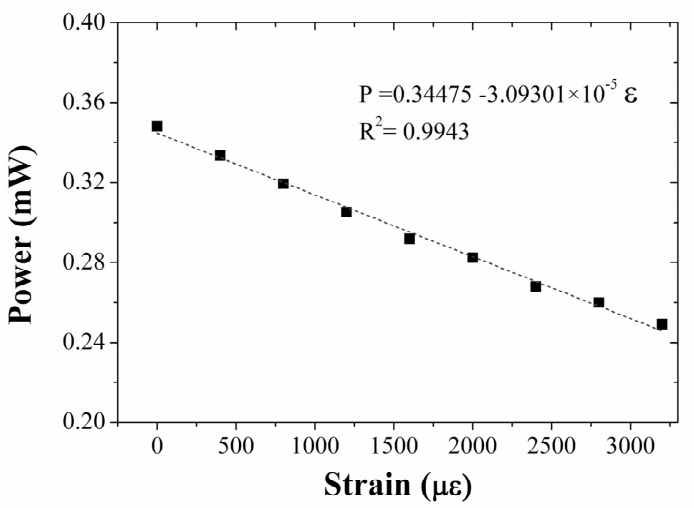

Fig. 9. Relationship between the fiber laser peak power and strain applied to the LPG (FBG was kept steady at room temperature).

After the completion of the performance evaluation of individual sensitivities, a study of the combined sensor response to temperature/strain variations was carried out. It is important to stress that the aim of this test was to assure the proper response of the laser when both the laser wavelength and power variation occurred due to changes in the two sensors simultaneously. Given the sensitivity of both FBG and LPG on strain and temperature and for ease of the implementation, in this work the power change induced by the LPG was controlled by the temperature variation and the shift of the FBG was controlled by strain variation. To achieve this, the LPG was heated in the climatic chamber meanwhile the FBG was strained using the translation stage. 
In the first test 63 samples were taken for both temperature and strain sensing using the LPG and the FBG respectively (Fig. 10). The temperature of the LPG was set between 25 and $85^{\circ} \mathrm{C}$ with a step change of $10{ }^{\circ} \mathrm{C}$ (no strain was applied to the LPG). For every temperature step a strain sweep was performed to the FBG centered at $\lambda=1537.3 \mathrm{~nm}$ from 0 to $3200 \mu \varepsilon$ with a step change of $400 \mu \varepsilon$ (FBG remained at room temperature).

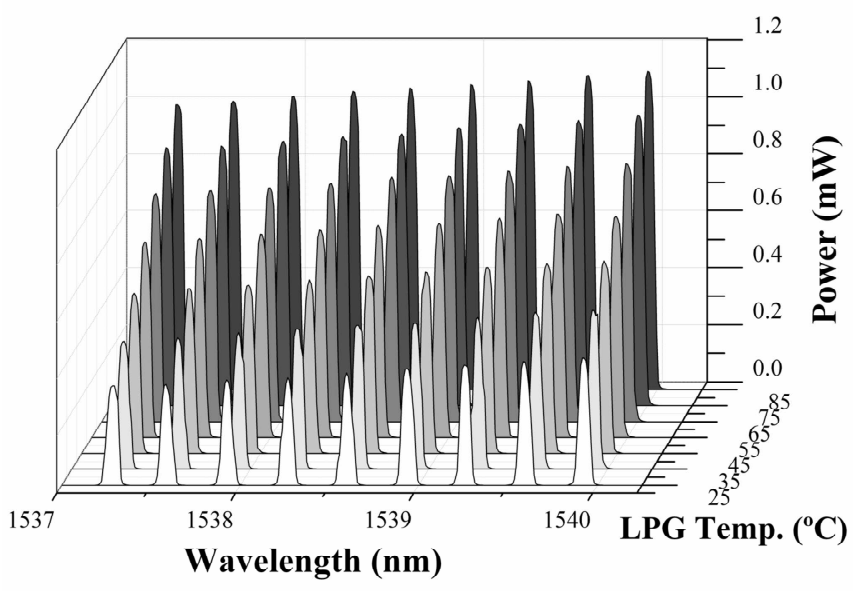

Fig. 10. Power and wavelength variation of the output signal when different temperature and strain was applied to the FBG and LPG respectively (no strain or temperature changes applied to the LPG and FBG respectively).

As shown in Fig.10, the wavelength and the power of the output signal change linearly with the strain applied to the FBG. Based on the experimental data obtained, the LPG sensitivity for strain $k_{\lambda \varepsilon}$ is $0.824 \mathrm{pm} / \mu \varepsilon$ and for temperature $k_{P T}$ $0.011 \mathrm{~mW} /{ }^{\circ} \mathrm{C}$, as it was expected.

However, even though the power variations for a fixed wavelength are linear, there is also an additional power growth with the wavelength increase. As can be seen in the Fig. 10 and 11 , as the wavelength of the laser increases because of a strain change in the FBG at a constant LPG temperature, the power of the emission line rises slightly too. This is due to the fact that a positive displacement of the laser wavelength in the linear slope of the LPG (because of a change in the FBG sensor) increases the power in the same manner as if a negative displacement of the LPG shape occurred due to a temperature or strain variation at the LPG.

Consequently, when the wavelength varies, the power varies as well apart from the variation related to the temperature increase at the LPG. This increment must be quantified and corrected in order to get a proper temperature reading from the sensor. This linear dependence was measured as $k_{c}=0.0369 \mathrm{~mW} / \mathrm{nm}$.

Because of this, as it is shown in Fig. 11 (a) if the peak power of the laser is represented for every strain (on the FBG) and temperature (on the LPG) value, a shift can be seen in the power measured for an identical temperature. However, after subtracting the correcting factor, a unique fit line can be used with a sensitivity of $k_{P T}=0.011 \mathrm{~mW} /{ }^{\circ} \mathrm{C}$ (Fig. 11 (b)).
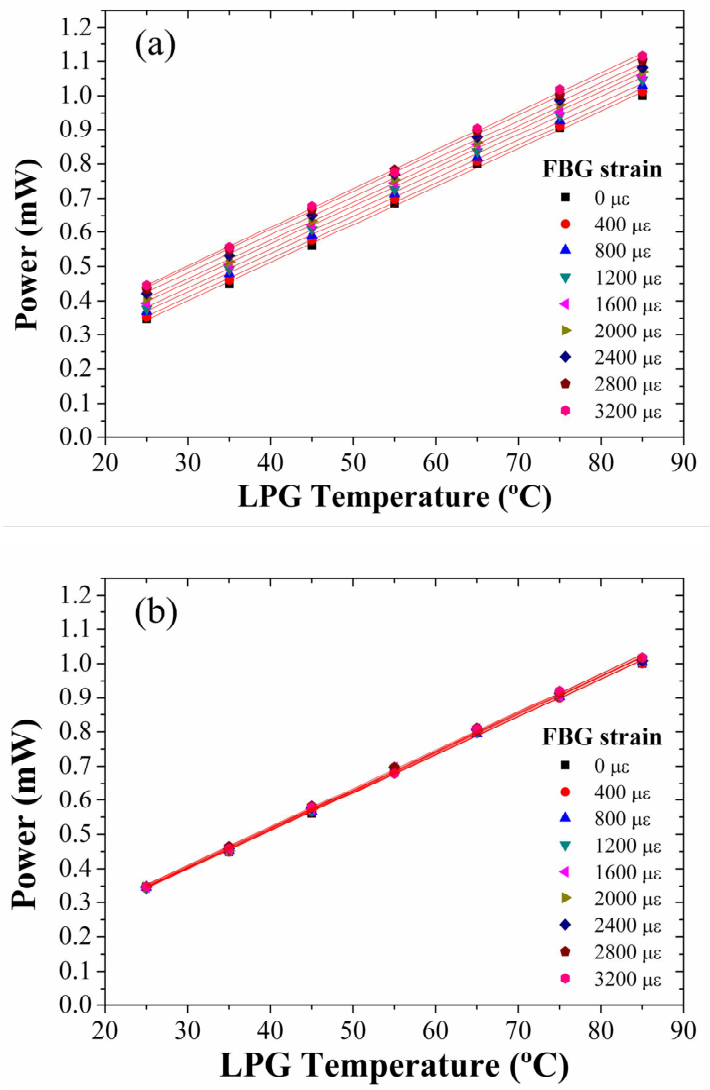

Fig. 11. Output power variation for different temperature and strain applied to the FBG and LPG respectively before (a) and after the correction (b).

At this point the sensitivities of the wavelength and the peak power of the emission line due to temperature and to strain variations can be measured. In addition, the linear behavior of the wavelength and peak power of the laser when both vary simultaneously has been observed as a function of physical changes at the LPG and FBG. Finally, the relationship between the sensitivities and the output signal has been studied when both sensor are subjected to identical strain and temperature values simultaneously.

The variation of the wavelength $(\Delta \lambda)$ and the peak power $(\Delta P)$ coupled out of the LPG as a function of the variation of the temperature $(\Delta T)$ and strain $(\Delta \varepsilon)$ measured can be written as follows:

$$
\begin{aligned}
& \Delta \lambda=\Delta T \cdot k_{\lambda T}+\Delta \varepsilon \cdot k_{\lambda \varepsilon} \\
& \Delta P=\Delta T \cdot k_{P T}+\Delta \varepsilon \cdot k_{P \varepsilon}+k_{c} \cdot \Delta \lambda
\end{aligned}
$$

Where $k_{\lambda \varepsilon}$ and $k_{\lambda T}$ are the laser wavelength sensitivities to strain and temperature respectively. Similarly $k_{P \varepsilon}$ and $k_{P T}$ are the laser peak power sensitivities to strain and to temperature. The power increase due to the wavelength shift is given by the correcting factor $k_{c}$

Equivalently, this relationship can be deducted from (1) and (2) and represented using a matrix form:

$$
\left(\begin{array}{c}
\Delta \lambda \\
\Delta P-k_{c} \Delta \lambda
\end{array}\right)=\left(\begin{array}{ll}
k_{\lambda T} & k_{\lambda \varepsilon} \\
k_{P T} & k_{P \varepsilon}
\end{array}\right) \cdot\left(\begin{array}{c}
\Delta T \\
\Delta \varepsilon
\end{array}\right)=[K] \cdot\left(\begin{array}{c}
\Delta T \\
\Delta \varepsilon
\end{array}\right)
$$


Accordingly the temperature and the strain are given by

$$
\begin{aligned}
\left(\begin{array}{c}
\Delta T \\
\Delta \varepsilon
\end{array}\right) & =\frac{1}{|K|}\left(\begin{array}{cc}
k_{P \varepsilon} & -k_{\lambda \varepsilon} \\
-k_{P T} & k_{\lambda T}
\end{array}\right) \cdot\left(\begin{array}{c}
\Delta \lambda \\
\Delta P-k_{c} \Delta \lambda
\end{array}\right)=\ldots \\
& =\frac{1}{k_{\lambda T} \cdot k_{P \varepsilon}-k_{\lambda \varepsilon} k_{P T}}\left(\begin{array}{cc}
k_{P \varepsilon}+k_{\lambda \varepsilon} k_{c} & -k_{\lambda \varepsilon} \\
-k_{P T}-k_{\lambda T} k_{c} & k_{\lambda T}
\end{array}\right)\left(\begin{array}{c}
\Delta \lambda \\
\Delta P
\end{array}\right)
\end{aligned}
$$

Finally, using the previously measured sensitivities, temperature and strain applied simultaneously to the sensors can be deducted from the laser wavelength and peak power as follows:

$$
\left(\begin{array}{c}
\Delta T \\
\Delta \varepsilon
\end{array}\right)=\left(\begin{array}{cc}
0.052 & 86.682 \\
121.303 & -943.617
\end{array}\right)\left(\begin{array}{c}
\Delta \lambda \\
\Delta P
\end{array}\right)
$$

Where wavelength shift $\Delta \lambda$ is expressed in nanometers $(\mathrm{nm})$, the peak power variation $\Delta P$ is in miliwatts $(\mathrm{mW})$, the strain variation $(\Delta \varepsilon)$ in microstrain $(\mu \varepsilon)$ and the temperature variation $(\Delta T)$ is expressed Celsius degrees $\left({ }^{\circ} \mathrm{C}\right)$.

To ensure the accuracy and repeatability of the measurements, it is important to assess the fiber laser power instability as the LPG sensor information is encoded in the amplitude of the signal obtained. Therefore, an instability study was carried out by measuring the output peak power once every minute until one hour (Fig. 12). This study shows that the peak power of the laser had very good power stability with an average variation of $1.31 \mu \mathrm{W}$ and a maximum variation of $3.17 \mu \mathrm{W}$ in one hour. Consequently, the peak power instability of the laser is not a limiting factor and the peak power of the laser can be used for sensing applications.

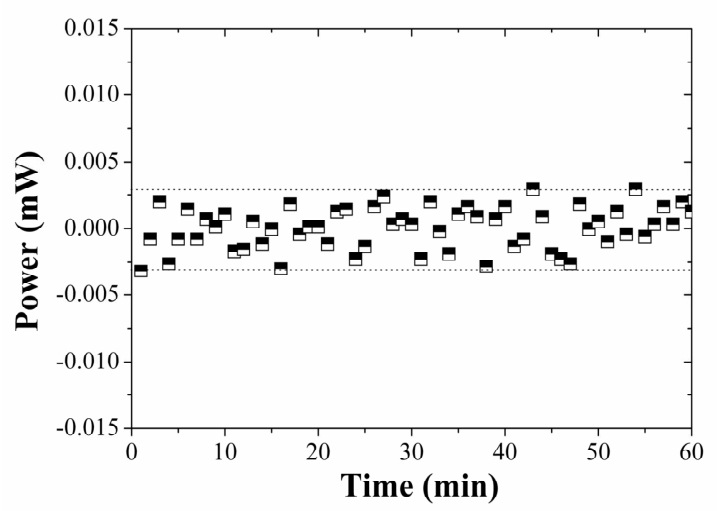

Fig 12. Power instability of the laser in one hour.

The same experimental process has been repeated multiple times and it is good to see that repeatable results have been obtained, showing a good repeatability of the measurements. In the final test, a FBG centered at $1536.2 \mathrm{~nm}$ was used to replace the FBG at $1537.2 \mathrm{~nm}$. The results agreed well with those obtained in the first test, although the measurement range and the constants used in the above equations were slightly different.

It is worth mentioning that once this technique has been validated, the results obtained can be easily adapted and improved by means of changing the gratings specifications. Since the sensing technique relies on the wavelength and the power of the laser, there are some key parameters of the gratings that must be taken into account for an appropriate design of the system. One of these parameters is the bandwidth of the LPG that determines the span of the linear slope in which the FBG must be constantly placed in order to get a correct reading. Therefore, the highest and the lowest possible wavelength of the emission line must be placed at the right and at the left limit of the linear slope of the LPG respectively in a worst case scenario. That is the reason why the Bragg wavelength of the FBG is also a critical parameter. In the proposed experiment, the FBG centered at $1537 \mathrm{~nm}$ was proved to stay within the linear part of the LPG shape from 0 $\mu \varepsilon$ and $25{ }^{\circ} \mathrm{C}$ (lowest emission wavelength, Fig. 3) to $3200 \mu \varepsilon$ and $90^{\circ} \mathrm{C}$ (highest emission wavelength). Another important parameter that is required to be considered for the design of the laser is the attenuation of the rejected band of the LPG. A higher attenuation implies an increase in the slope of the linear part of the LPG shape; consequently it increases the sensitivities related to the power $k_{P \varepsilon}$ and $k_{P T}$.

As a result, different gratings must be employed depending on the required accuracy and measuring range of temperature and strain. In this study the right slope of the LPG rejected band has been used to modulate the power of the laser but equally the left slope, with a different sensitivity, can also be utilized.

\section{CONCLUSION}

A new sensor system based on an erbium doped fiber laser has been presented and demonstrated. The temperature and strain information are included in the fiber laser wavelength and in the peak power level using a unique emission line. The fiber laser has an OSNR higher than $55 \mathrm{~dB}$ and a power instability of $3.17 \mu \mathrm{W}$ in one hour. The temperature and strain applied to the FBG shifts the wavelength of the laser with a sensitivity of $0.894 \mathrm{pm} / \mu \varepsilon$ and $8.97 \mathrm{pm} /{ }^{\circ} \mathrm{C}$ respectively. On the other hand the peak power of the laser increases linearly with the LPG temperature with a sensitivity of $0.0112 \mathrm{~mW} /{ }^{\circ} \mathrm{C}$ and also decreases linearly with the strain applied with a sensitivity of $-3.09 \times 10^{-5} \mathrm{~mW} / \mu \varepsilon$. Extensive tests have been undertaken in this work to verify the sensing principle, underpinning the system operation by applying physical variations to both sensors simultaneously. The power increase due to the wavelength shift has been also measured $\left(k_{c}=\right.$ $0.0369 \mathrm{~mW} / \mathrm{nm}$ ) and taken into account in the measurements. Finally equations have been derived for the effective calculation of the strain and the temperature as a function of the peak power and the wavelength of the emission line. The main advantage of this technique is that just one emission line is needed in order to monitor two parameters. This economic bandwidth usage enables multiplexing of a larger number of sensors within a certain optical bandwidth. Moreover, the high OSNR obtained allows the system to be used for remote sensing applications.

\section{ACKNOWLEDGMENT}

The authors would like to thank Liekki for providing the Erbium doped. 


\section{REFERENCES}

[1] H. Li, D. Li and G. Song, "Recent applications of fiber optic sensors to health monitoring in civil engineering," Engineering Structures, vol. 26, pp. 1647-1657, Sept. 2004.

[2] R. A. Perez-Herrera, A. Ullan, D. Leandro, M. Fernandez-Vallejo, M. A. Quintela, A. Loayssa, J. M. Lopez-Higuera and M. Lopez-Amo, "L-band multiwavelength single-longitudinal mode fiber laser for sensing applications," J. Lightwave Technol., vol. 30, pp. 1173-1177, 2012.

[3] M. Fernandez-Vallejo, M. Bravo and M. Lopez-Amo, "Ultra-long laser systems for remote fiber Bragg gratings arrays interrogation", IEEE Photonics Technology Letters, vol. 25 n.14 pp. 1362-1364, 2013.

[4] P. Peng, H. Tseng and S. Chi, "Long-Distance FBG Sensor System Using a Linear-Cavity Fiber Raman Laser Scheme," IEEE Photonics Technology Letters, vol. 16, pp. 575-577, 2004.

[5] A. M. Pinto and M. Lopez-Amo, "Photonic crystal fibers for sensing applications," Journal of Sensors, vol. 2012, Article ID 598178 (2012).

[6] D. Zhou, L. Wei, W. Liu and J. W. Y. Lit, "Simultaneous strain and temperature measurement with fiber Bragg grating and multimode fibers using an intensity-based interrogation method," IEEE Photonics Technology Letters, vol. 21, pp. 468-470, 2009.

[7] B. Dong, J. Hao, C.-Y. Liaw, B. Lin and S.C. Tjin, "Simultaneous strain and temperature measurement using a compact photonic crystal fiber inter-modal interferometer and a fiber Bragg grating", Applied Optics, vol. 49 (32), pp. 6232-6235, 2010.

[8] T. Li, X. Dong, C. Chiu Chan, L. Hu, W. Qian, "Simultaneous strain and temperature measurement based on a photonic crystal fiber modalinterference interacting with a long period fiber grating", Optics Communications, vol. 285, Issue 24, 1, Pages 4874-4877, Nov. 2012.

[9] B. Guan, H. Tam, X. Tao and X. Dong, "Simultaneous strain and temperature measurement using a superstructure fiber Bragg grating," IEEE Photonics Technology Letters, vol. 12, pp. 675-677, 2000.

[10] Z. Bai, W. Zhang, S. Gao, H. Zhang, L. Wang, Y. Liu, T. Yan, "Simultaneous measurement of strain and temperature using a long period fiber grating based on waist-enlarged fusion bitapers", Journal of Optics (United Kingdom), vol.16 (4), art. $\mathrm{n}^{\circ} .045401,2014$.

[11] W. Qiu, X. Cheng, Y. Luo, Q. Zhang, B. Zhu, "Simultaneous measurement of temperature and strain using a single bragg grating in a few-mode polymer optical fiber", Journal of Lightwave Technology, vol. 31 (14), art. $\mathrm{n}^{\circ}$. 6525393, pp. 2419-2425, 2013.

[12] L. Gao, L. Huang, L. Chen, X. Chen, "Simultaneous measurement of strain and temperature with a multi-longitudinal mode erbium-doped fiber laser" Optics Communications, vol. 297, pp. 98-101, 2013.

[13] Y.-N. Tan, Y. Zhang, L. Jin, B.-O. Guan, "Simultaneous strain and temperature fiber grating laser sensor based on radio-frequency measurement", Optics Express, vol. 19 (21), pp. 20650-20656, 2011.

[14] D. Leandro, M. Ams, M. López-Amo, T. Sun, K.T.V. Grattan, "Simultaneous measurement of strain and temperature using a unique LPG-coupled fibre laser scheme" Proceedings of SPIE - The International Society for Optical Engineering, vol. 9157, art. $\mathrm{n}^{\circ}$. 9157AB, 2014

[15] O. Duhem, M. Douay, "Effect of UV-induced birefringence on longperiod-grating coupling characteristics." Electronics Letters, 36 (5), pp. $416-417,2000$.

Daniel Leandro was born in Pamplona, Spain, in November 1984. He received the telecommunication engineering degree and communication master degree from the Universidad Publica de Navarra, Spain in 2010 and 2012, respectively. In 2012, he joined the Optical Communications Group, Department of Electrical and Electronic Engineering from Universidad Publica de Navarra. He has been a visiting Ph.D. student at the School of Engineering and Mathematical Sciences at City University of London. His research interests are in fiber optic lasers, optical amplifiers, optical fiber sensor networks and multiplexing architectures.

Martin Ams Dr Martin Ams received the B.Sc. degree in physics and the Ph.D. degree in optical laser physics from Macquarie University, Sydney, NSW, Australia, in 2001 and 2008, respectively. He then worked as a Research Associate at the MQ Photonics Research Centre and the Centre for Ultrahigh bandwidth Devices for Optical Systems (CUDOS), Macquarie University until mid-2011. He is currently a research Fellow at City University London. His research interests include laser fabrication of photonic waveguide devices and Bragg-gratings for use in telecommunication, sensing, structural monitoring, astronomy, quantum information and biophotonic applications. He has over eight years of photonics experience, is the author or co-author of 3 book chapters, 19 journal articles and 72 conference papers and is a regular referee for OSA, IEEE, IOP and AIP journals.

Manuel López-Amo (M'91, SM '98) was born in Madrid, Spain, in 1960. He received the telecommunications engineering degree and Ph.D. degrees from the Universidad Politécnica de Madrid, Spain in 1985 and 1989, respectively. From 1985 to 1996, he belonged to the Photonic Technology Department of the Universidad Politécnica de Madrid, where in 1990 he became an associate professor.

In 1996, he moved to Public University of Navarra (Pamplona, Spain) where he became a Full Professor in the Electrical and Electronic Engineering department and is currently the head of the optical communications group of this department. He has been Chairman of the Optoelectronic Committee of Spain. He has been leader of more than 30 research projects and he has coauthored more than 250 works in international refereed journals and conferences related with fiber-optic networks, optical amplifiers, fiber-optic sensors, and integrated optics. He is a member of the technical committees of the International Conference on fiber optic sensors (OFS), the European Workshop on optical fiber sensors (EWOFS), and European cost TD1001 action, among others. Professor López-Amo is senior member of the IEEE and member of the OSA.

Tong Sun received the B.Eng. degree, M.S. degree in engineering, and the $\mathrm{Ph} . \mathrm{D}$. degrees in engineering for work in mechanical engineering from the Department of Precision Instrumentation, Harbin Institute of Technology, Harbin, China, in 1990, 1993, and 1998, respectively. She also received the Ph.D. degree in applied physics from City University, London, U.K., in 1999. She was an Assistant Professor at Nanyang Technological University, Singapore, from 2000 to 2001, before she rejoined City University London, in 2001 , as a Lecturer. Subsequently, she was promoted to a Senior Lecturer in 2003, a Reader in 2006, and a Professor in 2008 at City University London. She has been leading a research team focused on developing a range of optical fiber sensors for a variety of industrial applications, including structural health monitoring, early fire detection, homeland security, process monitoring, food quality, and automotive emission monitoring, and has been working closely with partners across disciplines from academia and industry, both in the U.K. and overseas. She has authored or coauthored 230 scientific and technical papers. Prof. Sun is a member of the Institute of Physics and of the Institution of Engineering and Technology, and a Chartered Physicist and a Chartered Engineer, U.K.

Kenneth T. V. Grattan received the B.Sc. (Hons.) in physics and the Ph.D. degree in laser physics from the Department of Physics, Queen's University, Belfast, U.K., in 1974 and 1978, respectively, and the D.Sc. degree from City University, London, U.K., in 1992, for his sensor work. His research involved the use of laser-probe techniques for measurements on potential new laser systems. Following Queen's University, in 1978, he became a Research Fellow at the Imperial College of Science and Technology, London, U.K., sponsored by the Rutherford Laboratory to work on advanced photolytic drivers for novel laser systems. This involved detailed measurements of the characteristics and properties of novel laser species and a range of materials involved in systems calibration. In 1983, he joined City University, London, U.K. as a Lecturer in physics, and was appointed a Professor of Measurement and Instrumentation in 1991, and the Head of the Department of Electrical, Electronic and Information Engineering. The work has been sponsored by a number of organizations including EPSRC, the EU, DERA, private industry and BTG, with whom a joint patent for systems for monitoring in the water industry is held. He has authored or coauthored of more than 700 publications in major international journals and at conferences and is the coeditor (with Prof. B. T. Meggitt) of a five volume topical series on Optical Fiber Sensor Technology. His research interests include the use of fiber optic and optical systems in the measurement of a range of physical and chemical parameters. Prof. Gratton is extensively involved with the work of the professional bodies, having been Chairman of the Science, Education and Technology of the Institution of Electrical Engineers, the Applied Optics Division of the Institute of Physics, and was President of the Institute of Measurement and Control in 2000. He has been Deputy Editor of the Journal Measurement Science and Technology for several years and currently serves on the editorial board of several major journals in his field in the U.S. and Europe. In January 2001, he was appointed Editor of the IMEKO Journal Measurement and also serves on their General Council. He is also the Dean of the School of Informatics. Prof. Grattan received the Callendar Medal of the Institute of Measurement and Control in 1992, and the Honeywell Prize for work published in the Institute's journal 